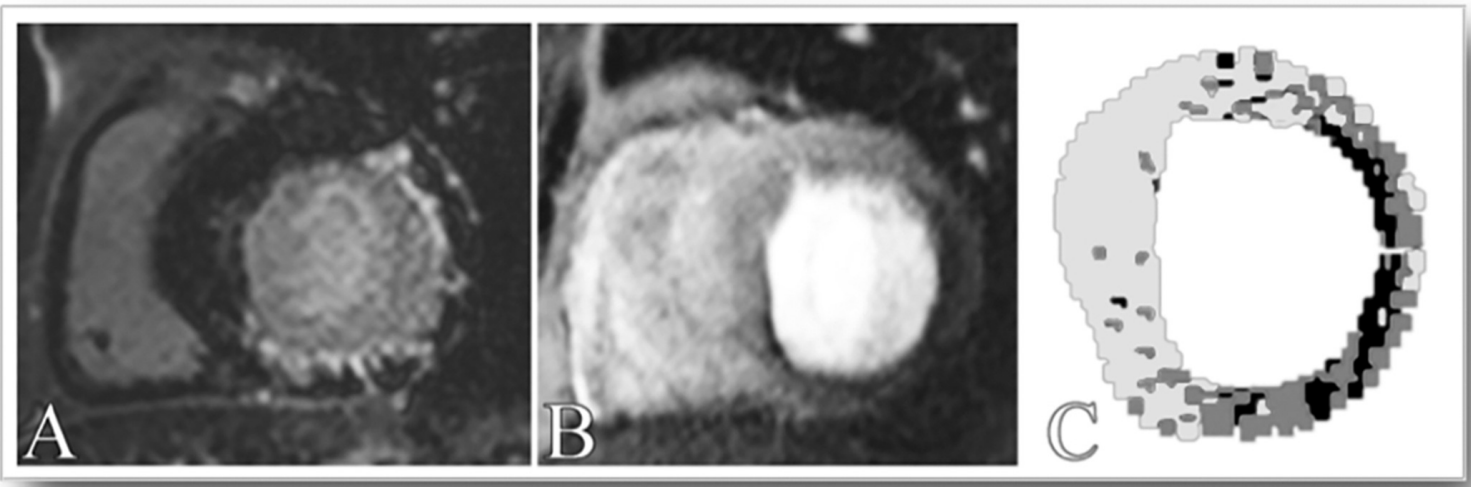

Abstract 23 Figure 1 Perfusion defect extending beyond the area of scar. A: LGE image shows a subendocardial scar involving the lateral and inferior wall; B: perfusion defect in the lateral and inferior wall; C: high-resolution perfusion map show area of scar (black), areas of ischaemia (dark grey) and normal areas of myocardium (light grey)

\section{ASSESSMENT OF THE EFFECTS OF TECHNIQUE ON PULMONARY ARTERIAL PULSE WAVE VELOCITY MEASUREMENT}

${ }_{1, *}$ R Weir-McCall, ${ }^{1} A$ Kamalasanan, 'DB Cassidy, ${ }^{1} A D$ Struthers, ${ }^{2} B J$ Lipworth, ${ }^{1} \mathrm{JG}$ Houston. ${ }^{1}$ Division of Cardiovascular and Diabetes Medicine, Medical Research Institute, University of Dundee, Dundee, UK; ${ }^{2}$ Scottish Centre for Respiratory Research, Medical Research Institute, University of Dundee, Dundee, UK

\subsection{6/heartjnl-2016-309668.24}

Aim The flow-area (QA) technique allows measurement of pulse wave velocity (PWV) from a single phase contrast slice. However in the pulmonary circulation reflected waves arrive during systole and may cause erroneous measurements using this technique. The aim of the study was to compare three post-processing calculations, one of which avoids the reflected wave, and the other which corrects for it, on the measurement of pulmonary PWV and its reproducibility.

Materials and methods 10 young healthy volunteers (YHV) (30\% male, mean age $31.5 \pm 7.6)$ and 20 older healthy volunteers (OHV) $(45 \%$ male, mean age $60.2 \pm 4.0)$ underwent MRI using phase contrast sequences through the main pulmonary artery (MPA), right pulmonary artery (RPA) and left pulmonary
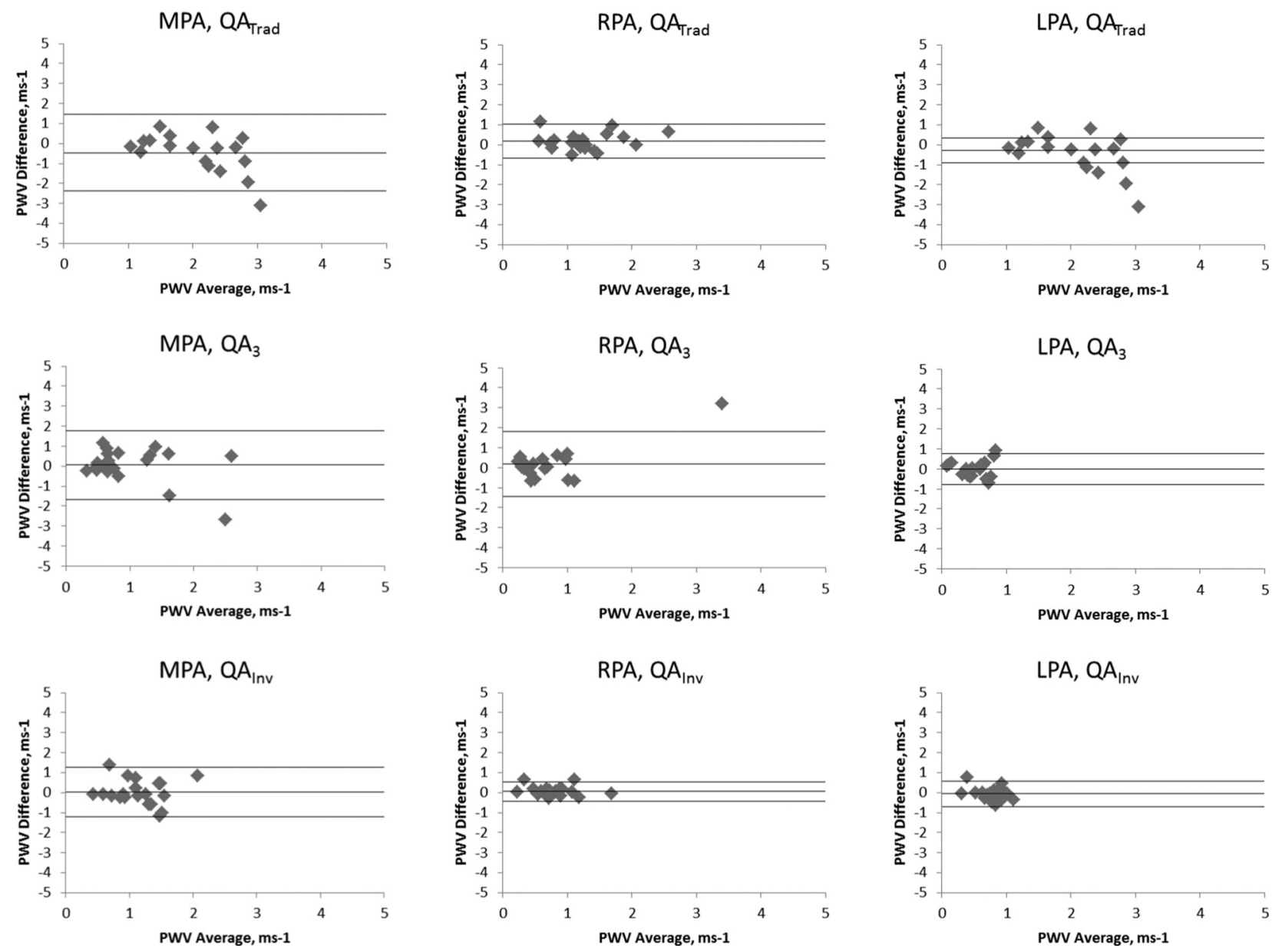

Abstract 24 Figure 1 Bland-Altman plots comparing interscan PWV repeatability using the 3 techniques in the 3 pulmonary arterial locations 
artery (LPA). Measurements were repeated at 6 months in the YHV cohort and on the same visit in the OHV cohort. QA PWV was calculated using three techniques: $\mathrm{QA}_{\text {Trad }}=\Delta \mathrm{Q} / \Delta \mathrm{A}$; $\mathrm{QA}_{3}=\Delta \mathrm{Q} / \Delta \mathrm{A}$ (using only the first three datapoints in the reflectionless upstroke); and $\mathrm{QA}_{\text {Inv }}=\sqrt{ }\left(\sum \Delta \mathrm{A}^{2} / \sum \Delta \mathrm{Q}^{2}\right)$.

Results $\mathrm{QA}_{\text {Trad }}$ produced significantly higher results than $\mathrm{QA}_{3}(\mathrm{p}$ $<0.001)$ and $\mathrm{QA}_{\text {Inv }}(\mathrm{p}<0.001)$, whilst there was no difference between $\mathrm{QA}_{3}$ and $\mathrm{QA}_{\text {Inv }}(\mathrm{p}=0.41)$. In scan-rescan reproducibility, $\mathrm{QA}_{\text {Inv }}$ yielded improved precision over $\mathrm{QA}_{\text {Trad }}$ and $\mathrm{QA}_{3}$ : mean (SD) of PWV differences $=-0.46(0.98) \mathrm{ms}^{-1}, 0.05(0.88) \mathrm{ms}^{-1}$, and $0.01(0.63) \mathrm{ms}^{-1}$ for the $\mathrm{QA}_{\text {Trad }}, \mathrm{QA}_{3}$, and $\mathrm{QA}_{\text {Inv }}$ of the MPA respectively; $0.17(0.43), 0.19(0.83)$ and $0.06(0.25) \mathrm{ms}^{-1}$ for the $\mathrm{QA}_{\text {Trad }}, \mathrm{QA}_{3}$, and $\mathrm{QA}_{\text {Inv }}$ of the $\mathrm{RPA}$ respectively; and $-0.29(0.31),-0.01(0.39)$ and $-0.06(0.32) \mathrm{ms}^{-1}$ for the $\mathrm{QA}_{\text {Trad }}$, $\mathrm{QA}_{3}$, and $\mathrm{QA}_{\text {Inv }}$ of the LPA respectively (see Figure 1).

Conclusion Calculations which account for wave reflections yield lower PWV than those that don't suggesting significant confounding effects from these early reflected waves. Combining a phase contrast sequence acquisition through the right pulmonary artery with a post processing technique to account for wave reflections yields the most reproducible measurements of pulmonary PWV. 